\title{
Isolated Trigeminal Neuropathy due to Trigeminal Nerve Root Hemorrhage
}

\author{
S. Almeida, C. Chalk, J. Minuk, R. Del Carpio, M. Guerin, and M. Levental
}

\begin{abstract}
Background: Isolated trigeminal neuropathy is uncommon; causes include trauma, inflammation, or neoplasm. Methods: We report a patient who fell and struck his head during a myocardial infarction, was treated with streptokinase, and developed symptoms and signs of an isolated trigeminal sensory neuropathy. Results: Imaging showed hemorrhage in the trigeminal nerve root; follow-up imaging showed resolution of the hemorrhage, but no underlying structural lesion. Conclusion: A combination of head trauma plus thrombolysis resulted in an isolated trigeminal neuropathy.
\end{abstract}

\begin{abstract}
RÉSUMÉ: Neuropathie isolée du trijumeau due à une hémorragie au niveau de la racine du trijumeau. Introduction: La neuropathie isolée du trijumeau est un problème rare causé entre autres par un traumatisme, de l'inflammation ou une néoplasie. Méthodes: Nous rapportons le cas d'un patient qui s'est frappé la tête en tombant au moment d'un infarctus du myocarde traité par streptokinase et qui a développé des symptômes et des signes de neuropathie sensorielle isolée du trijumeau. Résultats: L'imagerie a montré une hémorragie dans la racine du nerf trijumeau. Au cours du suivi, l'imagerie a montré une résolution de l'hémorragie sans lésion structurale sous-jacente. Conclusion: Une malencontreuse coïncidence de traumatisme crânien et de thrombolyse a provoqué une neuropathie isolée du trijumeau chez ce patient.
\end{abstract}

Can. J. Neurol. Sci. 1999; 26: 204-206

Isolated trigeminal neuropathy is uncommon. Identifiable causes include trauma, inflammation, neoplasm, or compression; sometimes no etiology can be found. ${ }^{1}$ We report an unusual case of isolated trigeminal neuropathy caused by a trigeminal nerve root hemorrhage.

\section{CASE Report}

A 62-year-old man with a history of smoking, gout, hypertension, hypercholesterolemia, and a remote myocardial infarction suddenly felt weak and short of breath while walking from his garden into his house. He abruptly lost consciousness and fell to the floor. His wife found him lying on the floor several minutes later and called an ambulance. When the ambulance arrived he was responsive, and he was taken to hospital. A left scalp laceration and a left buttock hematoma were noted, and an EKG showed ST segment elevation compatible with an anterior wall myocardial infarct. Intravenous streptokinase was given. Fourteen days later the patient underwent coronary artery bypass grafting.

The day after he was first admitted to the hospital, the patient reported numbness of the left face and gums, and of the left side of the tongue. He likened this to a dentist's freezing. The tactile quality of food was altered, but his taste sensation was intact. He denied visual disturbance, diplopia, facial pain or weakness, hearing changes, and motor or sensory disturbances in the limbs. Examination showed diminished cool and pinprick sensation affecting the entire left side of his face as far posteriorly as the vertex, but not including the angle of the jaw or the neck. The ear was spared with the exception of the tragus. Muscle power testing of the masseter, temporalis and pterygoids was normal. Eye movements, visual fields, optic fundi, and the tongue were normal, as was the remainder of his neurologic exam.

Cranial CT scan three days later revealed hemorrhage in the cisternal and intra-axial portion of the left trigeminal nerve, extending to the region of the principal trigeminal sensory nucleus (Figure 1). It caused a mild contour deformity of the left pons but no other significant mass effect. In the left parietotemporal region there was soft tissue swelling and a small scalp hematoma but no evidence of a skull fracture.

Magnetic resonance imaging (MRI) studies of the brain with and without gadolinium were performed two and eight months after the incident. On MPGR sequences at eight months, there was hypointense signal within the cisternal portions of the left trigeminal nerve, extending into the pons, corresponding to hemosiderin (Figure 2). No other structural lesion of the brainstem or trigeminal nerve was seen. Meckel's cave and the cavernous sinuses were unremarkable.

Neurophysiological testing seven months following his presentation demonstrated prolongation of the R1 latency and relative delay of the contralateral R2 response of the blink reflex when stimulating the left supraorbital nerve, consistent with conduction slowing in the left trigeminal nerve or root (Table 1). Concentric needle examination of the left masseter and facial nerve conduction studies were normal.

Eighteen months after onset, the patient's sensory symptoms and signs have improved only slightly.

\section{Discussion}

Several potential mechanisms could be responsible for our patient's trigeminal nerve root hemorrhage:

1. Trauma: There was clear clinical and radiological evidence that our patient struck his head on the ground when he fell. It seems likely that his brief loss of consciousness was caused by the head injury. Trigeminal sensory loss as a consequence of

From the Departments of Neurology and Neurosurgery (S.A., C.C., J.M.) and Radiology (R.D.C., M.G., M.L.) McGill University, Montréal.

RECEIVED DECEMBER 3, 1998. ACCEPTED IN FINAL FORM FEBRUARY 11, 1999. Reprint requests to: Dr. Colin Chalk, Division of Neurology, The Montréal General Hospital, 1650 Cedar Avenue, Montréal, Québec, Canada H3G 1A4 


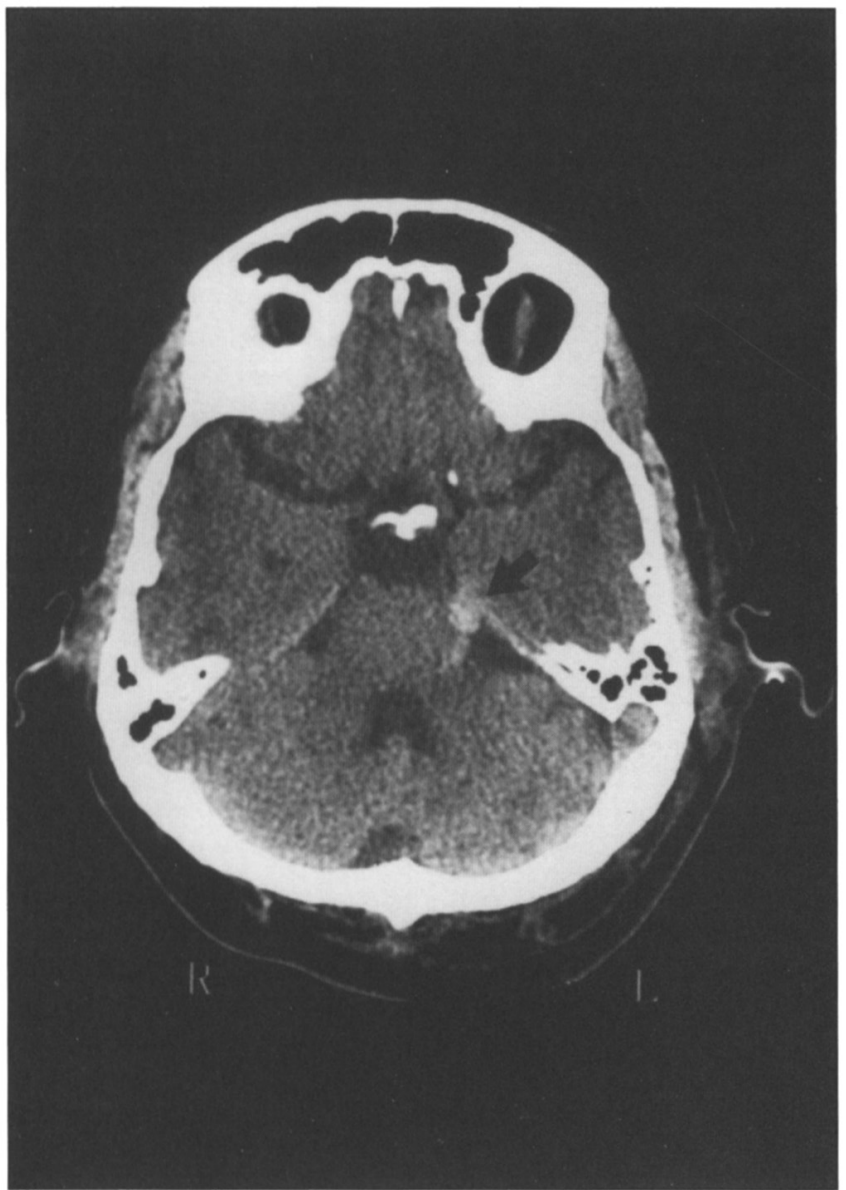

Figure 1: Cranial CT scan without contrast: The left trigeminal nerve (arrow) is enlarged and hyperdense (cisternal and intra-axial portions, extending to the region of the principal trigeminal sensory nucleus), consistent with hemorrhage within the nerve.

head injury is well recognized but uncommon (e.g., found in $4 \%$ in one series of 1800 head injuries ${ }^{2}$ ); usually it occurs in the setting of significant craniofacial trauma and is accompanied by other cranial neuropathies. Trigeminal nerve involvement is mostly due to injury to one or more superficial branches secondary to laceration, contusion or cranial fractures. The pre-ganglionic trigeminal nerve and Gasserian ganglion are well protected by their intimate anatomic relationship with the temporal bone and are seldom affected by trauma. Injury to these structures is most likely with penetrating wounds or fractures across the middle fossa and petrous ridge. ${ }^{2-7}$ However, even with basal skull fractures trigeminal nerve injury is rare. Cannon and Jahrsdoefer described 90 temporal bone fractures with none involving the trigeminal nerve, ${ }^{8}$ and Ghorayeb et al. described 82 temporal bone fractures with only one involving the trigeminal nerve. ${ }^{5}$

There are some reports of isolated trigeminal nerve involvement following trauma. A 22-year-old woman fell during a vasovagal attack and suffered a basal skull fracture and an isolated left trigeminal neuropathy. ${ }^{4}$ A young man who was struck on the left auriculotemporal area with a baseball bat developed signs of a basal skull fracture and a severe left sensorimotor trigeminal neuropathy; CT showed only left mastoid opacification. ${ }^{6}$

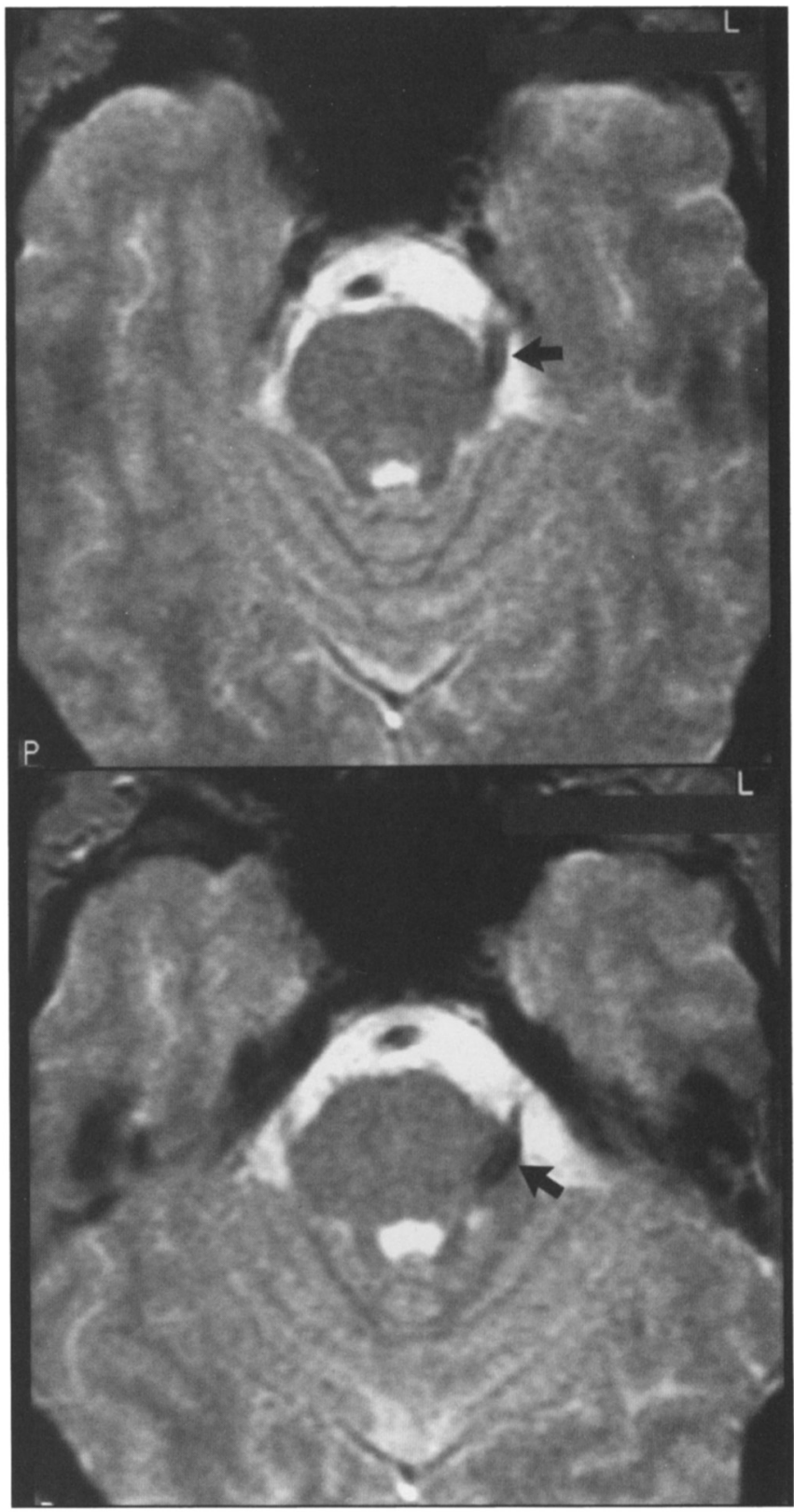

Figure 2: Axial MRI, MPGR sequence, 8 months after onset: hypointense signal within the cisternal portions of the left trigeminal nerve, extending into the pons, corresponding to hemosiderin (arrows). No other abnormalities were seen.

Although Aucott suggested that central trigeminal nerve injury should be considered a sign of basal skull fracture, ${ }^{4}$ our patient had no other clinical or radiological signs of basal skull fracture.

2. Trigeminal neuroma: A hemorrhagic mass in the trigeminal nerve raises the possibility of hemorrhage into a trigeminal neuroma, of which there are rare reports. ${ }^{9}{ }^{91}$ In one case, hemorrhage into a trigeminal neuroma followed a minor occipital head injury. ${ }^{10} \mathrm{An}$ underlying tumor was initially considered in our patient because of the slight mass effect seen on the first CT scan, but follow-up imaging revealed no tumor.

3. Vascular malformation: An occult vascular malformation could produce a small discrete hemorrhage, and might account 
Table: Blink responses, showing abnormal R1 latency and relative increase in contralateral R2 latency with stimulation on left, consistent with a left trigeminal neuropathy.

\begin{tabular}{|c|c|c|c|}
\hline Stimulation site & $\begin{array}{l}\text { Latencies (ms) } \\
\text { R1 ipsilateral }\end{array}$ & R2 ipsilateral & $\mathbf{R} 2$ contralateral \\
\hline L supraorbital nerve & 15 & 39 & 41 \\
\hline R supraorbital nerve & 13 & 35 & 32 \\
\hline Normals & $\leq 13$; side-to-side difference $<1.2$ & $\leq 41$ & $\leq 44 ;$ side-to-side difference $<8$ \\
\hline
\end{tabular}

for cases of pontine tegmental hemorrhage presenting as isolated trigeminal neuropathy." A patient with some resemblance to ours was reported by Veerapen et al. ${ }^{12}$ A 70 -year-old hypertensive woman presented with right facial numbness and gait unsteadiness of sudden onset. CT revealed a small hematoma in the brachium pontis with tracking of blood into the right trigeminal nerve root; at craniotomy, a hematoma was aspirated from the trigeminal nerve root and lateral pons. A vascular malformation was not seen, but neither MRI or angiography were performed. In our patient, the hemorrhage appears to have originated in the trigeminal nerve root, where a vascular malformation is unlikely, and follow-up MR studies showed only hemosiderin with no other signs of a vascular lesion. Although an occult vascular malformation cannot be entirely excluded, we have no firm basis to postulate such a lesion.

4. Streptokinase administration: Intracranial hemorrhage is an infrequent but well described complication of thrombolytic therapy for myocardial infarction. In the GUSTO-1 trial, for example, 246 of 41,021 patients suffered intracranial hemorrhage. ${ }^{13}$ Most of these hemorrhages were large, solitary, supratentorial and intraparenchymal. Only $3 \%$ were located in the brain stem, and none involved isolated cranial nerves. We are unaware of any reports of cranial nerve hemorrhage due to thrombolysis.

We speculate that our patient's trigeminal nerve root hemorrhage was produced by a combination of head injury followed by thrombolysis. Although he struck the left side of his head when falling, there seems to be no other obvious anatomical reason why the left trigeminal nerve should have been affected alone, and follow-up imaging has revealed no underlying lesion in the trigeminal nerve which might predispose it to bleed. One and a half years after the injury his recovery has been limited. This is probably because of axonal injury proximal to the Gasserian ganglion, where the non-schwannian myelin and extracellular milieu of the CNS will prevent any regeneration of the centrally directed trigeminal fibers.

\section{REFERENCES}

1. Hughes R. Diseases of the fifth cranial nerve. In: Dyck $\mathrm{P}$ and Thomas P, eds. Peripheral Neuropathy. 3rd ed. Philadelphia: W.B. Saunders, 1993: 801-817.

2. Hughes B. The results of injury to special parts of the brain and skull. In: Rowbotham GF, ed. Acute Injuries of the Head. London: Livingstone, 1964: 422-424.

3. Jefferson G, Schorstein J. Injuries of the trigeminal nerve, its ganglion and its divisions. Br J Surg 1955; 42: 561-581.

4. Aucott W. Central trigeminal injury: a sign of basal skull fracture. J R Coll Surg Edinb 1988; 33: 161-162.

5. Ghorayeb B, Yeakley J, Hall J, Jones B. Unusual complications of temporal bone fractures. Arch Otolaryngol Head Neck Surg 1987; 113: 749-753.

6. Schecter A, Anziska B. Isolated complete post-traumatic trigeminal neuropathy. Neurology 1990; 40: 1634 .

7. Summers C, Wirtschafter J. Bilateral trigeminal and abducens neuropathies following low-velocity, crushing head injury. J Neurosurg 1979; 50: 508-511.

8. Cannon C, Jahrsdoerfer R. Temporal bone fractures. Arch Otolaryngol 1983; 109: 285-288.

9. Ooi G, Peh W, Fung C. Case report: acute haemorrhagic presentation of trigeminal neuroma. Br J Radiol 1996; 69: 363-365.

10. Shimabukuro H, Masuzawa T, Miyagi K, Sato F. Trigeminal neurinoma revealed by intratumoral hemorrhage following a minor head injury. Surg Neurol 1983; 19: 346-350.

11. Holtzman $R$, Zablozki V, Yang W, Leeds N. Lateral pontine tegmental hemorrhage presenting as isolated trigeminal sensory neuropathy. Neurology 1987; 37: 704-706.

12. Veerapen R. Spontaneous lateral pontine hemorrhage with associated trigeminal nerve root hematoma. Neurosurgery $1989 ; 25: 451$ 454.

13. Gebel J, Sila C, Sloan M, et al. Thrombolysis-related intracranial hemorrhage: a radiographic analysis of 244 cases from the GUSTO-1 trial with clinical correlation. Stroke 1998; 29: 563569. 